\title{
THE UNIFORMIZATION OF A CLASS OF SIMPLY CONNECTED RIEMANN SURFACES
}

\author{
H. B. CURTIS, JR.
}

After defining a class of simply connected Riemann surfaces, this study determines the type of a surface from this class and obtains the forms of the uniformizing function and its derivative.

Definition of class of surfaces. Let $\left\{a_{n}\right\}_{n=1}^{\infty}$ and $\left\{b_{n}\right\}_{n=1}^{\infty}$ be two infinite sequences, and for each $n \geqq 1$ let $\left\{a_{i}(n)\right\}_{i=1}^{q_{n}}$ and $\left\{b_{i}(n)\right\}_{i=1}^{q_{n}}$ be finite sequences such that for $n \geqq 1$ and $1 \leqq i \leqq q_{n}-1$ :

$$
0<a_{i}(1)<b_{i}(1)<a_{i+1}(1)<b_{i+1}(1)<a_{1} ;
$$

for $n$ even and $n \geqq 2$,

$$
a_{n-1}<b_{n-1}<b_{i}(n)<a_{i}(n)<b_{i+1}(n)<a_{i+1}(n)<b_{n}<a_{n} ;
$$

for $n$ odd and $n \geqq 3$,

$$
b_{n-1}<a_{n-1}<a_{i}(n)<b_{i}(n)<a_{i+1}(n)<b_{i+1}(n)<a_{n}<b_{n} .
$$

A surface $F$ of the class to be discussed consists of sheets $S_{n}$ and $S_{i}(n), n=1,2,3, \cdots, i=1,2, \cdots, q_{n}$, over the $w$-sphere where, for $S_{n}$ and $S_{i}(n)$ copies of the $w$-sphere:

(a) $S_{1}$ is slit along the real axis from $a_{i}(1)$ to $b_{i}(1), i=1,2, \cdots, q_{1}$, and from $a_{1}$ to $b_{1}$. (b) for $n \geqq 1, S_{2 n}$ is slit along the real axis from $a_{2 n-1}$ to $b_{2 n-1}$, from $b_{2 n}$ to $a_{2 n}$, and from $b_{i}(2 n)$ to $a_{i}(2 n), i=1,2, \cdots$, $q_{2 n}$. (c) for $n \geqq 1, S_{2 n+1}$ is slit along the real axis from $b_{2 n}$ to $a_{2 n}$, from $a_{2 n+1}$ to $b_{2 n+1}$, and from $a_{i}(2 n+1)$ to $b_{i}(2 n+1), i=1,2, \cdots, q_{2 n+1}$. (d) for $n \geqq 1$ and $i=1,2, \cdots, q_{n}, S_{i}(n)$ is slit along the real axis between $a_{i}(n)$ and $b_{i}(n)$. (e) $S_{n}$ and $S_{n+1}$ are joined along the slits between $a_{n}$ and $b_{n}$ to form first order branch points at the end points. (f) $S_{i}(n)$ is joined to $S_{n}$ along the slits between $a_{i}(n)$ and $b_{i}(n)$ to form first order branch points at the end points.

A special case of this class was considered in [2]. However, only the type of a surface was established.

The uniformizing function. $F$ is simply connected, and by the Fundamental Mapping Theorem there exists a unique function $g$ which maps $F$ in a one-to-one way onto $|z|<R \leqq \infty$, and where for $f(z)=g^{-1}(z), f(0)=0 \in S_{1}$ and $f^{\prime}(0)=1$. By slitting each sheet of $F$ along the uncut part of the real axis, two surfaces of hyperbolic type are obtained, and an application of the reflection principle to the

Received by the editors August 26, 1959. 
uniformizing function of one of these surfaces in the upper half plane shows that $f(z)$ is real for $z$ real. Furthermore, the image of $F$ in the $z$-plane satisfies the following properties. The image of $S_{n}$ or $S_{i}(n)$ is a region symmetric about the real axis. $S_{1}$ and $S_{i}(1), i=1,2, \cdots$, $q_{1}$, are mapped onto a domain containing $z=0$ and bounded by a simple closed curve $C_{1}$ which intersects the real axis at $\alpha_{1}$ and $-\beta_{1}$. For $n>1, S_{n}$ and $S_{i}(n), i=1,2, \cdots, q_{n}$, are mapped onto an annular region about $z=0$ bounded by two simple closed curves $C_{n-1}$ and $C_{n}$ which intersect the real axis at $-\beta_{n-1}$ and $\alpha_{n-1}$, and $-\beta_{n}$ and $\alpha_{n}$, respectively. Each $S_{i}(n)$ is mapped onto a domain bounded by a simple closed curve $C_{i}(n)$ which intersects the real axis at $(-1)^{n+1} \beta_{i}(n)$ and $(-1)^{n+1} \alpha_{i}(n)$. If $(-1)^{n} \gamma_{n}$ and $(-1)^{n+1} \gamma_{i}(n)$ denote the image in the $z$-plane of $w=\infty$ for $S_{n}$ and $S_{i}(n)$, while $(-1)^{n} \delta_{n}$ and $(-1)^{n+1} \delta_{i}(n)$ denote the image of $w=0$ for $S_{n}$ and $S_{i}(n)$, respectively, then the following inequalities are satisfied:

for $n$ even, $n \geqq 2$,

$$
0<\alpha_{n-1}<\delta_{n}<\gamma_{n}<\alpha_{n}<\alpha_{n+1} ;
$$

for $n$ odd, $n \geqq 3$,

$$
0>-\gamma_{1}>-\beta_{1}>-\beta_{n-1}>-\delta_{n}>-\gamma_{n}>-\beta_{n}>-\beta_{n+1} ;
$$

for $1 \leqq i \leqq q_{1}-1$,

$$
0<\alpha_{i}(1)<\delta_{i}(1)<\gamma_{i}(1)<\beta_{i}(1)<\alpha_{i+1}(1)<\beta_{i+1}(1)<\alpha_{1} ;
$$

for $n$ even, $n \geqq 2,1 \leqq i \leqq q_{n}-1$,

$$
\begin{aligned}
-\beta_{n-1} & >-\beta_{i}(n)>-\delta_{i}(n)>-\gamma_{i}(n)>-\alpha_{i}(n)>-\beta_{i+1}(n) \\
& >-\alpha_{i+1}(n)>-\beta_{n} ;
\end{aligned}
$$

for $n$ odd, $n \geqq 3,1 \leqq i \leqq q_{n}-1$,

$$
\alpha_{n-1}<\alpha_{i}(n)<\delta_{i}(n)<\gamma_{i}(n)<\beta_{i}(n)<\alpha_{i+1}(n)<\beta_{i+1}(n)<\alpha_{n} .
$$

The approximating closed surfaces. Let $F_{n}$ be the part of $F$ formed from $S_{k}, k=1,2, \cdots, 2 n+1$, and $S_{i}(k), k=1,2, \cdots, 2 n$ and $i=1,2, \cdots, q_{2 k}$, with the slits in $S_{2 n+1}$ from $a_{2 n+1}$ to $b_{2 n+1}$ and from $a_{i}(n+1)$ to $b_{i}(2 n+1), i=1,2, \cdots, q_{2 n+1}$, deleted.

Notation.

$$
\begin{array}{rlrl}
\alpha_{\phi}^{*} & =1-z / \alpha_{\phi} . & & \alpha_{\phi}^{* *}(k)=1+(-1)^{k} z / \alpha_{\phi}(k) . \\
\beta_{\phi}^{*}=1+z / \beta_{\phi} . & \beta_{\phi}^{* *}(\mathrm{k})=1+(-1)^{k} z / \beta_{\phi}(k) . \\
\gamma_{\phi}^{*}=1-(-1)^{k} z / \gamma_{\phi} . & \gamma_{\phi}^{* *}(k)=1+(-1)^{k} z / \gamma_{\phi}(k) . \\
\delta_{\phi}^{*}=1-(-1)^{k} z / \delta_{\phi} . & \delta_{\phi}^{* *}(k)=1+(-1)^{k} z / \delta_{\phi}(k) .
\end{array}
$$


LEMMA 1.

$$
R_{n}(z)=\left[z / \gamma_{1, n}^{*}\right] \prod_{k=1}^{2 n}\left[\delta_{k, n}^{*} / \gamma_{k, n}^{*}\right] \prod_{k=1}^{2 n}\left[\prod_{i=1}^{q_{k}} \delta_{i, n}^{* *}(k) / \gamma_{i, n}^{* *}(k)\right] \delta_{2 n+1, n}^{*},
$$

where

$$
R_{n}^{\prime}(z)=\prod_{k=1}^{2 n}\left[\left(\alpha_{k, n}^{*} \beta_{k, n}^{*} / \gamma_{k, n}^{* 2}\right) \prod_{i=1}^{q_{k}} \alpha_{i, n}^{* *}(k) \beta_{i, n}^{* *}(k) / \gamma_{i, n}^{* *}(k)^{2}\right],
$$

defines a mapping of the z-plane onto $F_{n}$.

PROOF. $F_{n}$ is a simply connected closed surface and hence is the Riemann surface of the inverse of a unique rational function $R_{n}$ such that $w=R_{n}(z), R_{n}(0)=0 \in S_{1}, R_{n}^{\prime}(0)=1$, and $R_{n}(\infty)=\infty \in S_{2 n+1}$.

LEMMA 2. $F$ is parabolic.

Proof. Suppose that $F$ is not parabolic, and thus $g$ is a mapping of $F$ onto $\{|z|<R<\infty\}$. Let $D_{n}$ be the $z$-plane slit along the real axis from $\alpha_{2 n, 2 n}$ to $+\infty . \zeta=\psi_{n}(z)=g\left[R_{n}(z)\right]$ defines a schlicht mapping of $D_{n}$ onto a simply connected region $\Delta_{n}$ of the $\zeta$-plane bounded by $C_{2 n+1}, C_{i}(2 n+1), i=1,2, \cdots, q_{2 n+1}$, and the segments $\left(\alpha_{2 n}, \alpha_{2 n+1}\right)$ and $\left(-\beta_{2 n+1},-\gamma_{2 n}\right)$. Then $\alpha_{2 n, 2 n} \leqq d\left(0, C_{2 n+1}\right) \leqq R<\infty$ where $d\left(0, C_{2 n+1}\right)$ is the distance from $\zeta=0$ to the curve $C_{2 n+1}$, and thus there exists a subsequence $\left\{\alpha_{2 n_{i}, 2 n_{i}}\right\}$ such that $\alpha_{2 n_{i}, 2 n_{i}} \rightarrow A \leqq R$. Then $\psi_{n_{i}}$ is a schlicht mapping of $D_{n_{i}}$ into $\Delta_{n_{i}}$, where for $n_{i}$ sufficiently large, $\Delta_{n_{i}}$ $C\{\zeta|| \zeta \mid \leqq R\}$. If $D$ is the $z$-plane slit along the real axis from $z=A$ to $+\infty$, then $\left\{\psi_{n_{i}}\right\}$ forms a normal family by Montel's Theorem and $\psi_{n_{j}}(z) \rightarrow \psi(z)$ uniformly on any compact subset of $D . \psi$ maps $D$ schlichtly onto $\{\zeta|| \zeta \mid \leqq R\}$. Then $R_{n_{j}}(z) \rightarrow H(z)=f[\psi(z)]$, where $H$ is a function meromorphic in $D$. $H(z) \not \equiv \infty$ because $R_{n}(0)=0$.

Let $D^{*}$ be the $z$-plane slit along the real axis from $z=A$ to $-\infty$. Now $R_{n_{j}}(z)$ is defined in $D^{*}$ and for $j$ sufficiently large assumes no negative real values in any compact subset of $D^{*}$. Then $\left\{R_{n_{j}}\right\}$ is a normal family in $D^{*}$, so that a subsequence $\left\{R_{m}\right\}$ of $\left\{R_{n_{j}}\right\}$ converges uniformly on any compact subset of $D^{*}$ to a function $G$. Because of a common domain of convergence, $G$ is the analytic continuation of $H$. Then $w=G(z)$ defines a mapping of the $z$-plane punched at $z=A$ and $\infty$ one to one and conformally onto an open doubly connected Riemann surface $F^{*}$ of which $F$ is a subsurface obtained by inserting some slits in $F^{*}$ over the real axis. Clearly this is impossible, and thus $R=\infty$.

LEMMA 3. $R_{n}(z) \rightarrow f(z)$ uniformly on any compact subset of $|z|<\infty$ as $n \rightarrow \infty$. 
Proof. Because $F$ is parabolic and thus $\left\{\Delta_{n}\right\}$ converges in the sense of Carathéodory to its kernel $|\zeta|<\infty$, it follows that $z=R_{n}^{-1}[f(\zeta)] \rightarrow \zeta=g\left[R_{n}(z)\right]$ uniformly on any compact subset of $|\zeta|<\infty$. Then $\left\{D_{n}\right\}$ converges to $|z|<\infty$ and thus $R_{n}(z) \rightarrow f(z)$ uniformly on any compact subsets of $|z|<\infty$.

LEMmA 4. $\alpha_{k, n} \rightarrow \alpha_{k}, \quad \beta_{k, n} \rightarrow \beta_{k}, \quad \gamma_{k, n} \rightarrow \gamma_{k}, \quad \delta_{k, n} \rightarrow \delta_{k}, \quad \alpha_{i, n}(k) \rightarrow \alpha_{i}(k)$, $\beta_{i, n}(k) \rightarrow \beta_{i}(k), \gamma_{i, n}(k) \rightarrow \gamma_{i}(k)$, and $\delta_{i, n}(k) \rightarrow \delta_{i}(k)$ as $n \rightarrow \infty$.

Proof. This is a consequence of Hurwitz's Theorem.

Lemma 5. The infinite product

$$
\pi(z)=\left[z / \gamma_{1}^{*}\right]\left[\prod_{k=2}^{\infty} \delta_{k}^{*} / \gamma_{k}^{*}\right] \prod_{k=1}^{\infty}\left[\prod_{i=1}^{q_{k}} \delta_{i}^{* *}(k) / \gamma_{i}^{* *}(k)\right]
$$

converges uniformly on any compact subset of $|z|<\infty$.

Proof. $\pi(z)$ can be decomposed onto four infinite products similar to $M(z)=\prod_{k=1}^{\infty} \delta_{2 k}^{*} / \gamma_{2 k}^{*}$. Since $F$ is parabolic and $\left\{\Delta_{n}\right\} \rightarrow\{|\zeta|<\infty\}$, then $\delta_{k} \rightarrow \infty$ and $\gamma_{k} \rightarrow \infty$ as $k \rightarrow \infty$. Therefore, for any $R>0$ there exists $n_{0}=n_{0}(R)$ such that $\delta_{k}>R$ and $\gamma_{k}>R$ for $k \geqq n_{0}$. Then consider $M_{p}(z)=\prod_{k=n_{0}}^{n_{0}+p} \delta_{2 k}^{*} / \gamma_{2 k}^{*}$. $M_{p}$ is holomorphic and $M_{p}(z) \neq 0$ for $|z| \leqq R$. A sufficient condition for the uniform convergence of $M_{p}$ in a region as $p \rightarrow \infty$ is the uniform convergence of

$$
\sum_{k=n_{0}}^{n 0+p} \log \left(\delta_{2 k}^{*} / \gamma_{2 k}^{*}\right)
$$

in this region as $p \rightarrow \infty$, where the logarithm is the principal value. By the Cauchy criterion, this last series converges uniformly in $\{|z| \leqq R\}$ provided for $\epsilon>0$ and for all $z$ such that $|z| \leqq R$ there exists $N(\epsilon)>0$ such that for $n>N(\epsilon)$ and for all $p>0$,

$$
\left|\sum_{k=n_{0}+n}^{n_{0}+n+p} \log \left(\delta_{2 k}^{*} / \gamma_{2 k}^{*}\right)\right|<\epsilon .
$$

Because $\cdots \delta_{2 k}<\gamma_{2 k}<\delta_{2 k+2}<\gamma_{2 k+2}, \cdots$, then for $m \geqq 1$ and $p>0$,

and thus

$$
0<\sum_{k=n_{0}+n}^{n++n+p}\left[\left(1 / \delta_{2 k}\right)^{m}-\left(1 / \gamma_{2 k}\right)^{m}\right]<\left(1 / \delta_{2 n_{0}+2 n}\right)^{m}
$$

$$
\begin{array}{r}
\left|\sum_{k=n_{0}+n}^{n++n+p} \log \left(\delta_{2 k}^{*} / \gamma_{2 k}^{*}\right)\right|=\left|\sum_{m=1}^{\infty}\left[z^{m} / m\right]\left\{\sum_{k=n_{0}+n}^{n{ }^{n+n+n}+p}\left[\left(1 / \delta_{2 k}\right)^{m}-\left(1 / \gamma_{2 k}\right)^{m}\right]\right\}\right| \\
\leqq \sum_{m=1}^{\infty}\left(R^{m} / m\right)\left(1 / \delta_{2 n_{0}+2 n}\right)^{m} \leqq \sum_{m=1}^{\infty}\left(R / \delta_{2 n_{0}+2 n}\right)^{m}=R /\left(\delta_{2 n_{0}+2 n}-R\right) .
\end{array}
$$


Because $\delta_{k} \rightarrow \infty$ as $k \rightarrow \infty$, the required inequality is established. Hence $M(z)$ converges uniformly on any compact subset of the z-plane. Similarly, the other products into which $\pi(z)$ can be decomposed converge uniformly on any compact subset of the z-plane.

Lemma 6. $\pi(z)=f(z)$.

Proof. Because of Lemma 4 , there exists $r>0$ such that $R_{n}(z) / z \neq 0$ and $\pi(z) / z \neq 0$ for $|z|<r$. Because for $m \geqq 1$,

$$
0<\sum_{k=i}^{\infty}\left[\left(1 / \delta_{2 k}\right)^{m}-\left(1 / \gamma_{2 k}\right)^{m}\right] \leqq\left(1 / \delta_{2 i}\right)^{m}
$$

and

$$
0<\sum_{k=i}^{\infty}\left[\left(1 / \delta_{2 k, n}\right)^{m}-\left(1 / \gamma_{2 k, n}\right)^{m}\right]<\left(1 / \delta_{2 i, n}\right)^{m},
$$

it follows with the aid of Lemma 4 that for $n_{0}>1$,

$$
\begin{aligned}
0 & \leqq \limsup _{n \rightarrow \infty}\left|\sum_{k=1}^{n}\left[\left(1 / \delta_{2 k, n}\right)^{m}-\left(1 / \gamma_{2 k, n}\right)^{m}\right]-\sum_{k=1}^{\infty}\left[\left(1 / \delta_{2 k}\right)^{m}-\left(1 / \gamma_{2 k}\right)^{m}\right]\right| \\
& \leqq \limsup _{n \rightarrow \infty} \mid \sum_{k=n_{0}}^{n}\left[\left(1 / \delta_{2 k, n}\right)^{m}-\left(1 / \gamma_{2 k, n}\right)^{m}\right]-\sum_{k=n_{0}}^{\infty}\left[\left(1 / \delta_{2 k}\right)^{m}-\left(1 / \gamma_{2 k}\right)^{m} \mid\right. \\
& <\lim _{n \rightarrow \infty}\left|\left(1 / \delta_{2 n_{0}, n}\right)^{m}+\left(1 / \delta_{2 n_{0}}\right)^{m}\right|=2 /\left(\delta_{2 n_{0}}\right)^{m} .
\end{aligned}
$$

Since $\delta_{2 n_{0}} \rightarrow \infty$ as $n_{0} \rightarrow \infty$,

$$
\lim _{n \rightarrow \infty}\left\{\sum_{k=1}^{n}\left[\left(1 / \delta_{2 k, n}\right)^{m}-\left(1 / \gamma_{2 k, n}\right)^{m}\right]-\sum_{k=1}^{\infty}\left[\left(1 / \delta_{2 k}\right)^{m}-\left(1 / \gamma_{2 k}\right)^{m}\right]\right\}=0 .
$$

Thus in this manner all the coefficients of the Taylor expansion of $\log \left[R_{n}(z) / \pi(z)\right]$ about $z=0$ can be shown to have a limit of zero as $n \rightarrow \infty$, and thus $\lim _{n \rightarrow \infty} \log \left[R_{n}(z) / \pi(z)\right]=0$ which implies that $\lim _{n \rightarrow \infty} R_{n}(z)=f(z)=\pi(z)$.

Lemma 7. The infinite product

$$
P(z)=\prod_{k=1}^{\infty}\left\{\alpha_{k}^{*} \beta_{k}^{*} /\left(\gamma_{k}^{*}\right)^{2}\right\}\left\{\prod_{i=1}^{q_{k}} \alpha_{i}^{* *}(k) \beta_{i}^{* *}(k) /\left[\gamma_{i}^{* *}(k)\right]^{2}\right\}
$$

converges uniformly on any compact subset of the z-plane.

Proof. $P(z)$ can be decomposed into four infinite products similar to $Q(z)=\prod_{k=1}^{\infty} \alpha_{2 k}^{*} \alpha_{2 k+1}^{*} /\left(\gamma_{2 k+2}^{*}\right)^{2}$. Because for $m \geqq 1$ and $p>0$,

$$
0<\sum_{k=n_{0}+n}^{n++n+p}\left[\left(1 / \alpha_{2 k}\right)^{m}+\left(1 / \alpha_{2 k+1}\right)^{m}-2 /\left(\gamma_{2 k+2}\right)^{m}\right]<2 /\left(\alpha_{2 n_{0}+2 n}\right)^{m},
$$


an application of the Cauchy criterion similar to that described in the proof of Lemma 5 establishes the convergence of $Q(z)$. Similarly, the other factors of $P(z)$ converge.

Lemma 8. $P(z)=f^{\prime}(z)$.

Proof. Because for all $i>0$ and $m \geqq 1$,

$$
\begin{aligned}
& 0<\sum_{k=i}^{n}\left(1 / \alpha_{2 k}\right)^{m}+\sum_{k=i}^{n-1}\left(1 / \alpha_{2 k+1, n}\right)^{m}-\sum_{k=i}^{n-1} 2 /\left(\gamma_{2 k+2, n}\right)^{m}<2 /\left(\alpha_{2 i, n}\right)^{m}, \\
& 0<\sum_{k=i}^{\infty}\left[\left(1 / \alpha_{2 k}\right)^{m}+\left(1 / \alpha_{2 k+1}\right)^{m}-2 /\left(\gamma_{2 k+2}\right)^{m}\right]<2 /\left(\alpha_{2 i}\right)^{m},
\end{aligned}
$$

and similar inequalities, it follows in a manner similar to the proof of Lemma 6 that $\lim _{n \rightarrow \infty} \log \left[R_{n}^{\prime}(z) / P(z)\right]=0$ and thus $P(z)=f^{\prime}(z)$.

These lemmas prove the following theorem.

A Riemann surface of the class defined is parabolic and its mapping function $f$ is given by

$$
f(z)=\left[z / \gamma_{1}^{*}\right] \prod_{k=2}^{\infty}\left[\delta_{k}^{*} / \gamma_{k}^{*}\right] \prod_{k=1}^{\infty}\left[\prod_{i=1}^{q_{k}} \delta_{i}^{* *}(k) / \gamma_{i}^{* *}(k)\right]
$$

where

$$
f^{\prime}(z)=\prod_{k=1}^{\infty}\left\{\left[\alpha_{k}^{*} \beta_{k}^{*} /\left(\gamma_{k}^{*}\right)^{2}\right]\left[\prod_{i=1}^{q_{k}} \alpha_{i}^{* *}(k) \beta_{i}^{* *}(k) /\left(\gamma_{i}^{* *}(k)\right)^{2}\right]\right\} .
$$

The $\alpha, \beta, \gamma$, and $\delta$ are ordered as indicated at the beginning of the paper.

It should be noted that the factors in the infinite products in Lemmas 5 and 7 are quotients. Hence the representations in this theorem are products of quotients, not quotients of products.

\section{REFERENCES}

1. G. R. MacLane, Riemann surfaces and asymptotic values associated with real entire functions, The Rice Institute Pamphlet, 1952.

2. H. E. Taylor, Determination of the type and properties of the mapping functions for certain classes of Riemann surfaces, Ph.D. Thesis, The Rice Institute, 1950.

The Rice Institute AND

The University of TeXas 